\title{
Precancerous colorectal lesions (Review)
}

\author{
VINCENZA CONTEDUCA $^{1}$, DOMENICO SANSONNO ${ }^{2}$, SABINO RUSSI $^{2}$ and FRANCO DAMMACCO ${ }^{2}$ \\ ${ }^{1}$ Department of Medical Oncology, IRCCS Istituto Scientifico Romagnolo per lo Studio e \\ la Cura dei Tumori (I.R.S.T.), Meldola (FC); ${ }^{2}$ Department of Internal Medicine and \\ Clinical Oncology, University of Bari ‘Aldo Moro’, Medical School, Bari, Italy
}

Received March 25, 2013; Accepted May 3, 2013

DOI: 10.3892/ijo.2013.2041

\begin{abstract}
Colorectal cancer (CRC) is a common and often lethal tumor. Over the last 25 years, remarkable progress has been made in understanding its biological and molecular features and in elucidating the steps involved in colon carcinogenesis. This, in turn, has led to a more rational and effective clinical approach to the treatment of CRC. While colorectal adenoma is the most frequent precancerous lesion, other potentially premalignant conditions, including chronic inflammatory bowel diseases and hereditary syndromes, such as familial adenomatous polyposis, Peutz-Jeghers syndrome and juvenile polyposis, involve different sites of the gastrointestinal tract with an overall incidence of less than 5\%. In all such cases, disease recognition at an early stage is essential to devise suitable preventive cancer strategies. These topics are addressed in this review, along with the most important epidemiological, pathogenetic and clinical features that lead to malignant transformation. Novel biomarkers for early cancer prediction, detection, prognostic evolution, and the response to treatment are critically assessed as well. Continued improvements in our knowledge of the molecular basis of CRC and
\end{abstract}

Correspondence to: Dr Vincenza Conteduca, Department of Medical Oncology, IRCCS Istituto Scientifico Romagnolo per lo Studio e la Cura dei Tumori (I.R.S.T.), Via Piero Maroncelli 40, 47014 Meldola (FC), Italy

E-mail: cinzia.conteduca@libero.it

Abbreviations: APC, adenomatous polyposis coli; BAX, Bcl2associated $\mathrm{X}$ protein; CIMP, $\mathrm{CpG}$ island methylator phenotype; $\mathrm{CIN}$, chromosomal instability; COX-2, cyclo-oxygenase-2; CRC, colorectal cancer; EGFR, epidermal growth factor receptor; EpCAM, epithelial cell adhesion molecule; FAP, familial adenomatous polyposis; 5-FU, 5-fluorouracil; HNPCC, hereditary non-polyposis colon cancer; IGF-1, insulin-like growth factor-1; IL, interleukin; JPS, juvenile polyposis syndrome; MAPK, mitogen-activated protein kinase; MMR, mismatch repair; MSI, microsatellite instability; MUTYH, mutY homologue; MAP, MUTYH-associated polyposis; PGE2, prostaglandin E2; PI3K, phosphatidylinositol 3-kinase; TGF- $\beta$, transforming growth factor- $\beta$; VEGF, vascular endothelial growth factor

Key words: adenoma, colorectal cancer, intestinal polyps, malignant transformation, precancerous lesions, tumor prevention the transfer of this information into daily clinical practice will reduce the burden of this disease.

\section{Contents}

1. Epidemiology

2. Pathogenesis

3. Diagnosis

4. Screening and surveillance

5. Treatment

6. Conclusion

\section{Epidemiology}

Colorectal cancer (CRC) is a tumor that develops from the progression of acquired or hereditary premalignant lesions. It arises from interactions among different risk factors (environmental, dietary, familial and hereditary) that become relevant during the different stages of colorectal carcinogenesis. CRC is a major public health problem worldwide, although in developed countries survival rates have significantly improved over the past two decades, reflecting continuous progress in our understanding of its biology, epidemiology, prevention, early diagnosis and treatment. Nonetheless, CRC remains the third most commonly diagnosed cancer and the third leading cause of cancer death in both men and women, with over 1.2 million new cases and over 600,000 deaths expected in $2013(1,2)$.

As for esophageal and gastric cancer $(3,4)$, various pathological conditions or a positive personal or family history that predisposes to CRC development are associated with a particular frequency and lifetime risk of disease occurrence. Thus, individuals are considered to be at average, increased or high risk. About $70 \%$ of CRCs are sporadic, perhaps attributable to unidentified genetic factors in the context of dietary and environmental factors. Individuals 50 years of age or older are at average risk, with a $5 \%$ lifetime risk of developing CRC. The risk increases to $10-15 \%$ in individuals with a personal history of adenoma/sessile serrated polyps, inflammatory bowel disease, or a positive family history of CRC (5).

Colorectal adenoma. The precursors of almost all sporadic CRCs are colorectal adenomas. These typically asymptomatic lesions are often found incidentally during colonoscopy 
Table I. Features of colorectal polyps.

\begin{tabular}{lllll}
\hline Polyp type & Side & Size & Histological features & Risk of carcinoma
\end{tabular}

Conventional adenoma $(85-90 \%)$

$\begin{array}{lll}\text { Left colon } & \text { Variable } & \text { Tubular } 25 \% \\ \text { and rectum } & \text { Tubulo-villous } 15 \% \\ & \text { Villous } 5 \% \\ & \text { Variable grade of dysplasia }\end{array}$

Serrated adenoma $(10-15 \%)$

Hyperplastic

polyp (80-90\%)

Left colon $\quad<5 \mathrm{~mm}$

and rectum

Traditional serrated

adenoma (1-5\%)

Left colon

and rectum

Variable

Right colon $>5 \mathrm{~mm}$

Sessile serrated

adenoma (15-20\%)

Mixed polyp (1\%)
Right colon Variable
Slightly protruding;

no cytological atypia or

architectural dysplasia

\section{Often pedunculated;}

presence of dysplasia and/or

foci of intraepithelial neoplasia

Sessile;

no cytologic dysplasia

Combinations of conventional adenomas with different grades of dysplasia and serrated lesions

\section{Low risk (1\%): \\ $<1 \mathrm{~cm} / \leq 2$ polyps/tubular/ \\ low grade dysplasia \\ High risk (30-50\%): \\ $\geq 1 \mathrm{~cm} />3$ polyps/villous/ \\ high grade dysplasia}

None

The same risk as adenomatous polyps

Risk present, but degree not known

Degree of risk variable performed for unrelated symptoms or for CRC screening. At least $25 \%$ of men and $15 \%$ of women who undergo colonoscopic screening have one or more adenomas. Colorectal adenomatous polyps develop in up to $40 \%$ of people over the age of 60 (6). Although not all colonic polyps are adenomas and more than $90 \%$ of adenomas do not progress to cancer, a differential diagnosis that takes into account the various types of colorectal polyps and the accurate identification of those that will progress to cancer remain challenging (Table I). Some easily identifiable but wide-ranging pathological features, such as size, architectural growth, type, and dysplastic grade and organization, are predictive both of the natural history of these lesions and of the time frame of their potential evolution from adenoma to carcinoma.

The transformation rate of adenomatous polyps into carcinoma is about $0.25 \%$ per year. The size of the adenoma is a relevant determinant, given that cancer devlops in $1 \%$ of adenomas $<1 \mathrm{~cm}$, in $10 \%$ of adenomas $>1 \mathrm{~cm}$ and $<2 \mathrm{~cm}$, and in $50 \%$ of adenomas $>2 \mathrm{~cm}$. The histological features that determine the malignant potential of an adenoma are its growth pattern and grade of dysplasia. In adenomas with a mainly villous architectural configuration (tubulovillous and villous) and high-grade dysplasia, the risk of malignant transformation rises to $50 \%$ (5).

Between 10 and $15 \%$ of sporadic CRCs are likely to originate from serrated polyps, which have a significant malignant potential. Serrated polyps include hyperplastic polyps, which account for $80-90 \%$ of cases, but also sessile serrated adenomas, traditional serrated adenomas, and mixed polyps displaying features of both serrated and 'classical' adenomas.

Adenomatous polyps, particularly those with villous components, are regarded as precursors of CRC, unlike hyperplastic polyps. The term 'serrated adenoma' was used to describe a group of polyps sharing mixed features of hyperplastic polyps and adenomas. Serrated adenocarcinoma is a distinct variant of CRC, accounting for about $7.5 \%$ of all colorectal tumors and up to $17.5 \%$ of the most proximal colorectal tumors. Sessile serrated adenomas have some features of serrated adenomas, but their sessile configuration distinguishes them from their more pedunculated counterparts, i.e., traditional serrated adenomas.

Sessile serrated adenomas, traditional serrated adenomas, and conventional adenomas differ in their malignant potential, reflecting differences in the molecular pathways of carcinogenesis. Histological assessment has shown a significantly lower degree of high-grade dysplasia and carcinoma in situ in serrated adenomas than in traditional adenomas. Thus, serrated adenomas are less likely to develop into CRC than traditional adenomas, but the degree of risk is not yet known (7). However, recent studies have suggested a close association between the peculiar molecular features of mucinous carcinomas (i.e., higher diploidy index, lower expression of p53, more frequent DNA replication errors leading to microsatellite instability, specific codon 12 KRAS mutations) and sessile serrated adenomas. Mucinous histological features are often seen in sessile serrated polyps that progress to invasive adenocarcinoma, both in sporadic cases of CRC and in individuals with a well-defined genetic predisposition (8).

The risk of cancer from a colorectal adenoma is eliminated with its complete removal even if discovery of the adenoma indicates the possible risk of metachronous lesions with variable malignant potential according to endoscopic and histologic features. The presence of three or more colorectal adenomas or one or more advanced adenomas is associated with a risk of metachronous adenomas that is two to five times higher than in the general population. Other characteristics of the baseline adenoma (e.g., a location proximal to the splenic flexure) or of 
the patient (e.g., male sex, older age or a first-degree relative with CRC) are also considered predictive of metachronous adenomas or CRC. The overall risk of developing metachronous adenomas after the removal of an adenoma is about $5-10 \%$ per year $(9,10)$.

Chronic inflammatory bowel disease. Patients with a chronic inflammatory bowel disease (IBD), such as ulcerative colitis and Crohn's disease, are at greater risk of developing CRC. The risk of CRC increases with the extent and duration of the IBD. Thus, $15 \%$ of patients with a 30 -year history of ulcerative colitis will eventually develop CRC (11).

Familiarity. Patients with a first-degree relative (parent, sibling or offspring) who has had CRC have a two to three times higher risk of developing CRC than individuals with no family history. If the diagnosis in the relative was made at a young age or if more than one relative is affected, the risk is three to six times higher than in the general population. In general, about $25 \%$ of all CRC patients have a close relative who was diagnosed with the disease (12).

\section{Genetic syndromes}

Susceptibility to CRC is higher in individuals with well-defined rare genetic syndromes, which comprise about $5 \%$ of all cases of colorectal tumors. Thus, in patients with familial adenomatous polyposis (FAP) not surgically treated, the lifetime CRC risk is as high as $100 \%$ (Fig. 1). In addition, there is a greater risk of developing other malignancies (13).

Lynch syndrome. Also known as hereditary non-polyposis CRC (HNPCC), Lynch syndrome accounts for $2-4 \%$ of all CRC cases (14). Although individuals with HNPCC are predisposed to several types of cancer, the lifetime risk of CRC is the highest $(\sim 75 \%)$. Colon cancers and polyps arise in Lynch syndrome patients at a younger age than in the general population with sporadic neoplasias, and the tumors develop at a more proximal location. Histologically, the cancers are often poorly differentiated, mucinous and are infiltrated by large numbers of lymphocytes (15).

Familial adenomatous polyposis. With a prevalence of 1 in 10,000 individuals, FAP is the second most common genetic syndrome predisposing to CRC. For these individuals, the lifetime risk of CRC without prophylactic colectomy approaches $100 \%$. The characteristic features of FAP include the development of hundreds to thousands of colonic adenomas beginning in early adolescence. The average age of CRC diagnosis (if untreated) in FAP patients is 40 years; $7 \%$ develop the tumor by the age of 20 and $95 \%$ by the age of 50 . Attenuated FAP is a less severe form of the disease, with an average lifetime risk of CRC of 70\%. In this group, approximately 30 adenomatous polyps develop in the colon, colonic neoplasms tend to be located in the proximal colon, and cancer occurs at an older age. Other rare variants of FAP are Gardner's syndrome and Turcot's syndrome. The former is characterized by prominent extra-colonic features: epidermoid cysts, osteomas, dental abnormalities and/or desmoid tumors. The latter includes patients with colorectal adenomatous polyps; these patients are prone to developing malignant tumors of the central nervous system, above all medulloblastoma (16).

MUTYH-associated polyposis, Peutz-Jeghers syndrome, juvenile polyposis syndrome. These frequent genetic condi-

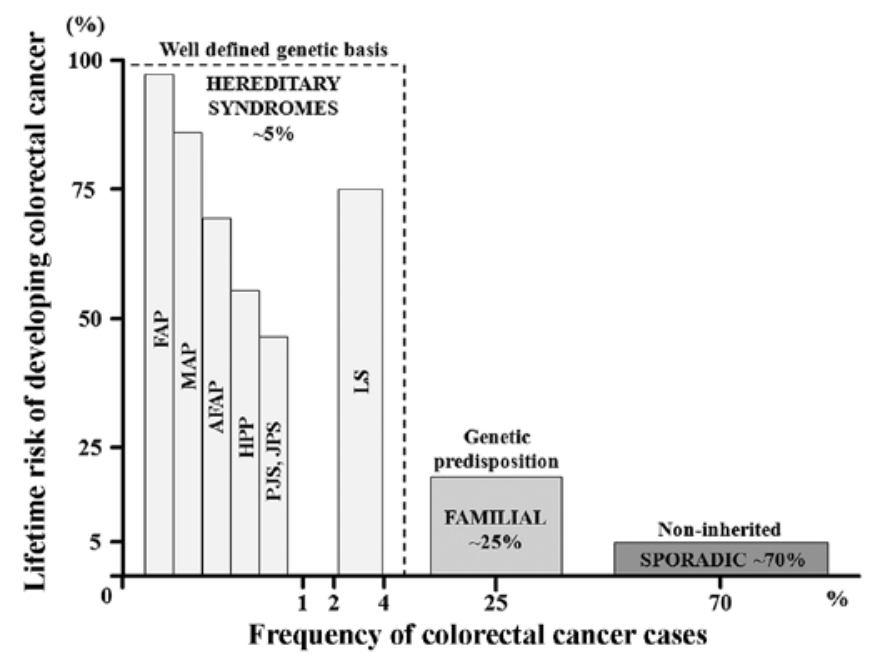

Figure 1. Proposed model of colon cancer risk susceptibility. Colon cancer risk can be stratified by the estimated lifetime risk of colon cancer (y-axis) versus the approximate frequency of colorectal cancer (CRC) cases ( $\mathrm{x}$-axis). The well-defined rare genetic conditions include high lifetime risk syndromes such as FAP (familial adenomatous polyposis coli), AFAP (attenuated FAP), MAP (MUTYH-associated polyposis), LS (Lynch syndrome), HPP (hyperplastic polyposis), and the hamartomatous polyp syndromes PJS (Peutz-Jeghers syndrome) and JPS (juvenile polyposis syndrome). The clinically defined 'familial subset' consists of individuals at increased risk based on a clear hereditary component, as determined from the family history, but with undefined causative genetic factors. The largest subset, accounting for $70 \%$ of CRC cases, comprises individuals in the general population with sporadic tumors. In this group, the lifetime risk derives from combinations of unidentified rare and common genetic factors in which environmental influences are likely to play a role.

tions have an incidence of $<1 \%$ and their characteristics include distinct cancer risks, clinical features and genetic patterns. Patients with MUTYH-associated polyposis (MAP) develop adenomatous polyposis of the colorectum and have an $80 \%$ risk of CRC. The colonic phenotype of this syndrome is similar to that of attenuated FAP, including a propensity for developing proximal colonic neoplasms (17). Peutz-Jeghers and juvenile polyposis syndromes are hamartomatous conditions associated with an increased risk for colorectal and other malignancies. In Peutz-Jeghers patients, the most consistent extra-colonic feature is a muco-cutaneous pigmentation typically occurring in childhood and seen on the lips, oral mucosa, and periorbital area. The typical gastrointestinal lesions are histologically distinctive hamartomatous polyps (96\% of cases) that arise in the small bowel. Gastric and colonic polyps develop in approximately 25 and $30 \%$ of these patients, respectively. The lifetime risk of gastrointestinal cancer is $40 \%$ (18). The main features of juvenile polyposis syndrome are multiple polyps, most prominently in the colon but also in the stomach, duodenum, and small bowel, that develop in young patients. As in Peutz-Jeghers syndrome, the lifetime risk of CRC is approximately $40 \%$ (19).

Hyperplastic polyposis. Little is known about the etiology, natural history, and incidence of this rare condition, which is characterized by multiple and/or large hyperplastic polyps of the colon. These patients have a $>50 \%$ risk of developing CRC before the age of 50-60 years. The tumors tend to develop in the proximal colon and metachronous and synchronous cancers are frequently observed (20). 


\section{Pathogenesis}

Geographic and dietary factors. Geographic differences in CRC rates among immigrant populations over time suggest that diet and lifestyle strongly influence the CRC risk. High-level consumption of red and/or processed meat increases the risk of both colon and rectal cancer (21). CRC also has been linked to even moderate alcohol consumption, with an increased risk of $23 \%$ (22). However, the precise role of specific dietary elements in colorectal tumorigenesis is poorly understood.

Metabolic alterations. Obesity, especially abdominal obesity, is associated with a higher risk of colorectal adenoma and CRC, especially in men. This association implies that obesity promotes the early stages of carcinogenesis but it may also play a role in the growth of advanced adenomas, both of which favor adenoma recurrence. The mechanistic relationship between obesity and colon cancer risk is not well established but may include the mitogenic properties of insulin, obesity-related insulin resistance, and associated hyperinsulinemia. Insulin could also promote colorectal carcinogenesis by increasing the levels of bioactive insulin-like growth factor (IGF)-1, either directly or through a decrease of IGF binding protein levels, which leads to increased free IGF-1. Obesity may be a pro-inflammatory state, as demonstrated by the high systemic levels of proinflammatory cytokines, chemokines, and other acute phase proteins released from adipose tissue, which consists not only of adipocytes but also of immune cells (23).

Individuals with diabetes mellitus have an increased risk of CRC. This may be related to the metabolic consequences of obesity, physical inactivity, and insulin resistance, but the evidence supporting a link between hyperglycemia and CRC is inconsistent (24).

Sex hormones. Differences in sex hormones might explain the lower female/male ratio in the population under than above the age of 55 years (i.e., in pre-menopausal vs. post-menopausal women). This likely reflects the inverse relation between tumor progression and the expression of type $\beta$ estrogen receptors on colon cancer cells and thus the inhibitory effect of estrogens on tumor growth. Estrogens have been suggested to alter bile acid composition, modulate colonic transit, reduce the production of mitogenic IGF-1, and stimulate the expression of the mismatch repair (MMR) gene MLH1 in colonic epithelial cells (25).

Chronic inflammation. As discussed above, chronic inflammation is a key risk factor for CRC in patients with IBDs. The risk of colon cancer increases not only with disease duration and the anatomic extent of the colitis but also with the presence of other inflammatory disorders (such as primary sclerosing cholangitis), whereas it decreases in patients taking anti-inflammatory agents (such as steroids). Inflammatory cells produce reactive oxygen and nitrogen species, which can alter the expression of genes encoding carcinogenesis-related factors (p53, DNA MMR proteins, and DNA base excision-repair proteins), transcription factors (nuclear factor- $\mathrm{\kappa B}$ ) or signaling proteins (cyclo-oxygenase-2, COX-2). Moreover, individual components of the innate and adaptive immune response (immune cells, cytokines, chemokines and the intestinal bacterial flora) have been implicated in carcinogenesis, via genetic or epigenetic alterations $(26,27)$. Thus, there is a close relationship between colonic inflammation and neoplasia, which develops progressively: no dysplasia $\rightarrow$ indefinite dysplasia $\rightarrow$ low-grade dysplasia $\rightarrow$ high-grade dysplasia $\rightarrow$ carcinoma.

There are also similarities between the pathways of sporadic cancers and colitis-associated cancers, including the development of aneuploidy (chromosomal instabilities, CIN), microsatellite instabilities (MSI), DNA methylation, activation of the oncogene K-ras, activation of COX-2, and the mutation and eventual loss of heterozygosity of p53, adenomatous polyposis coli (APC), and two candidate tumor suppressor genes, namely deleted in colorectal carcinoma and deleted in pancreatic carcinoma 4 (DCC/DPC4). However, the frequency and sequence of these events differ between these CRC types (26).

Genetic and epigenetic instabilities. Genetic and epigenetic instabilities are a hallmark of colorectal carcinogenesis. The former comprises genomic instabilities, i.e., CIN and MSI, and the latter the cytosine-phosphate-guanine $(\mathrm{CpG})$ island methylator phenotype (CIMP) and global DNA hypo-methylation (28). Genomic instability characterizes the early steps of malignant transformation from adenoma into carcinoma, increasing the mutation rate and thereby facilitating the progression to malignancy. The most common form of genomic instability are CIN, which are found in about $85 \%$ of CRCs. CIN are recognized by the presence of aneuploidy, i.e., numerical chromosome changes, or multiple structural aberrations. They are an efficient mechanism for causing the physical loss of a wild-type copy of a tumor-suppressor gene such as APC, p53, and Short-name Mothers Against Decapentaplegic (SMAD) family member 4 (29).

MSI, which account for $15 \%$ of sporadic CRCs, are an epiphenomenon of the inactivation of genes involved in the repair of base-base mismatches in DNA, defined as MMR genes (hMSH2, hMLH1,hPMS1,hPMS2 or hMSH6). The loss of MMR function disrupts the ability of the affected cell to repair strand slippage within repetitive DNA sequence elements. Consequently, the sizes of the mononucleotide or dinucleotide repeats (microsatellites) interspersed throughout the genome are altered and tumor-suppressor genes whose functional regions contain these repeat sequences, such as those encoding transforming growth factor- $\beta$ (TGF- $\beta$ ) receptor type II and Bcl2-associated X protein (BAX), are inactivated. In MMR deficiency, the inactivation can be inherited, as occurs in HNPCC (about 95\% of the mutations involve hMSH2 or hMLH1), or acquired, as observed in tumors with methylation-associated silencing of a gene encoding an MMR protein, for example, biallelic silencing of the promoter region of the MLH1 gene by promoter methylation $(13,30)$. Recently, germline deletions in the EpCAM (epithelial cell adhesion molecule) gene, also known as TACSTD1, were found in a subset of families with Lynch syndrome in whom MMR gene mutations were absent because of the uncommon hypermethylation of the hMSH2 promoter (31).

Microsatellite status has been divided into three types, with different clinical and prognostic features with respect to CRC: i) microsatellite stable, with no instability; ii) low-level instability (MSI-low), with <30\% instability; and iii) high-level instability (MSI-high), with $>40 \%$ of the microsatellite loci showing instability. MSI-high occurs in more than $90 \%$ of patients with an 
inherited predisposition for CRC but in only $15 \%$ of those with sporadic CRCs, i.e., without familial predisposition.

Sporadic MSI tumors are associated with the serrated adenoma neoplasia pathway and frequently carry BRAF V600E mutations, which are not seen in patients whose cancers result from germline mutations in MMR genes (Lynch syndrome). Thus, the presence of a BRAF mutation in an MSI tumor effectively excludes the possibility of Lynch syndrome (32).

In patients with MAP, there is germline inactivation of a base excision repair gene, the mutY homologue (MUTYH, also called MYH). The MYH protein excises from DNA the 8-oxoguanine product of oxidative damage to guanine. Two MAP-associated mutations in MYH have been identified, Y179C and G396D, which together account for 85\% of the cases of this disease. By contrast, somatic inactivation of $\mathrm{MYH}$ has not been detected in sporadic CRC (17).

Epigenetic instabilities in precancerous lesions and CRC are manifested as hyper-methylation of gene promoters containing CpG islands (CIMP) and global DNA hypo-methylation. CIMP is observed in about $50 \%$ of premalignant colonic adenomas and in about $50 \%$ of CRCs, which suggests that it is an early event in colorectal tumorigenesis. MSI are also detected in about $45 \%$ of CIMP-positive CRCs but in $100 \%$ of CIMP-positive cancers in which hMLH1 is methylated (33). The strong association between BRAF V600E mutations and CIMP CRC points to a role for activated BRAF in the pathogenesis of the methylator phenotype as well as a link between sporadic MSI and CIMP (34).

In addition to genomic and epigenomic instabilities, the accumulation of mutations in specific genes (tumor-suppressor genes or proto-oncogenes) is another pathogenetic route in the neoplastic progression of precancerous lesions to CRC. Thus, the malignant transformation of colon epithelial cells and thus CRC can arise from the loss/inactivation of tumor-suppressor genes that are not involved in specific signaling pathways, such as p53, and from recurrent cytogenetic aberrations, such as in the $18 \mathrm{q}$ loss of heterozygosity gene (28). Moreover, in CRC one or more cellular pathways may become deregulated. Of these, the best studied are the Wnt- $\beta$-catenin, TGF- $\beta$, epidermal growth factor receptor (EGFR), Ras/Raf/mitogen-activated protein kinase (MAPK) and phosphatidylinositol 3-kinase (PI3K) signaling pathways.

Mutations in the APC gene itself are predominantly associated with the classic tubular adenoma pathway and with CIN-type cancer (35). They occur in up to $70 \%$ of sporadic CRCs and explain the predisposition of patients with FAP to cancer development. The APC gene is located on chromosome $5 q 21$. Although germline-inactivating mutations can occur throughout the gene, somatic mutations are clustered in the mutation cluster region, between codons 1286 and 1513. The conventional form of the APC gene contains 15 exons. Exon 15 is the largest, comprising more than $75 \%$ of the 8,535 base pairs of coding sequence. In the tumors of FAP patients, it is the site of most germline and somatic mutations (36). In addition to the conventional form of APC, alternatively expressed exons of the gene encode protein isoforms, including truncated proteins. Interestingly, mutational analyses in FAP patients have identified significant genotype-phenotype correlations: i) severe polyposis ( $>5,000$ polyps), associated with mutations between codons 1250 and 1464; ii) attenuated polyposis (<100 polyps), in which mutations occur at the extreme $5^{\prime}$ and $3^{\prime}$ ends of the APC gene; iii) congenital hypertrophy of the retinal epithelium, associated with mutations between codons 457 and 1444; and iv) desmoid tumors, in which mutations are found between codons 1403 and 1578 (37).

In contrast to the truncating APC gene mutations implicated in FAP, APC I1307K is a single-nucleotide substitution that results in a non-truncating, missense mutation and thus to a single amino acid difference in the approximately 3,000 amino acids that constitute the APC protein. The APC I1307K variant is carried by an estimated $6 \%$ of the Ashkenazi Jewish population and its presence approximately doubles the risk of developing colorectal polyps and CRC in heterozygous carriers (38). Hyper-methylation of the APC promoter is an alternative mechanism for APC gene inactivation; it occurs in $18 \%$ of primary colorectal carcinomas and adenomas (39). Defects in the Wnt signaling pathway are an initiating event in $\mathrm{CRC}$ and underlie many preneoplastic lesions. Wnt signaling occurs when the oncoprotein $\beta$-catenin binds to nuclear LEF-1 and so creates a transcription factor that regulates genes involved in cellular activation. The $\beta$-catenin degradation complex regulates $\beta$-catenin levels by proteolysis. A component of this complex, the APC protein, not only degrades $\beta$-catenin but also inhibits its nuclear localization. The most common mutation in CRC inactivates the gene that encodes the APC protein. In the absence of functional APC, which acts as a brake on the $\beta$-catenin pathway, Wnt signaling is inappropriately and constitutively activated (40). Activating mutations in the $\beta$-catenin gene (CTNNB1) protect the encoded protein from APC-mediated degradation. CTNNB1 mutations are found more frequently in adenomas $(12.5 \%)$ than in invasive cancer (1.4\%), suggesting that CTNNB1-mutant tumors do not frequently progress to carcinoma (32).

Another early and critical step in adenoma development is the activation of prostaglandin signaling, especially the increased production of prostaglandin E2 (PGE2). In addition, growth factors, cytokines, inflammatory mediators and tumor promoters induce the overexpression of $\mathrm{COX}-2$ in about $43 \%$ of adenomas and $86 \%$ of carcinomas. COX-2 and PGE2 regulate proliferation, survival, migration and invasion in colorectal tumors. COX-2 also regulates angiogenesis, inducing the production of pro-angiogenic factors such as vascular endothelial growth factor (VEGF) and basic fibroblast growth factor, which may also contribute to the growth and lethal potential of CRC (28). PGE2 activity is increased by the loss of 15-prostaglandin dehydrogenase, the rate-limiting enzyme in PG degradation (41).

In summary, the accumulation of several acquired genetic and epigenetic changes transform normal glandular epithelial cells into invasive colorectal carcinoma. This stepwise transformation of normal epithelium into benign neoplasia (adenoma), followed by invasive carcinoma and eventually metastatic cancer, are described in the classic tumor progression model proposed by Fearon and Vogelstein (42). The complex progression to colorectal carcinogenesis also involves colon cancer-initiating stem cells $(43,44)$, located within the crypt unit, which account for $0.25-2.5 \%$ of the total number of tumor cells. An overview of the most important molecular alterations and the main risk factors in colorectal carcinogenesis is provided in Fig. 2. 

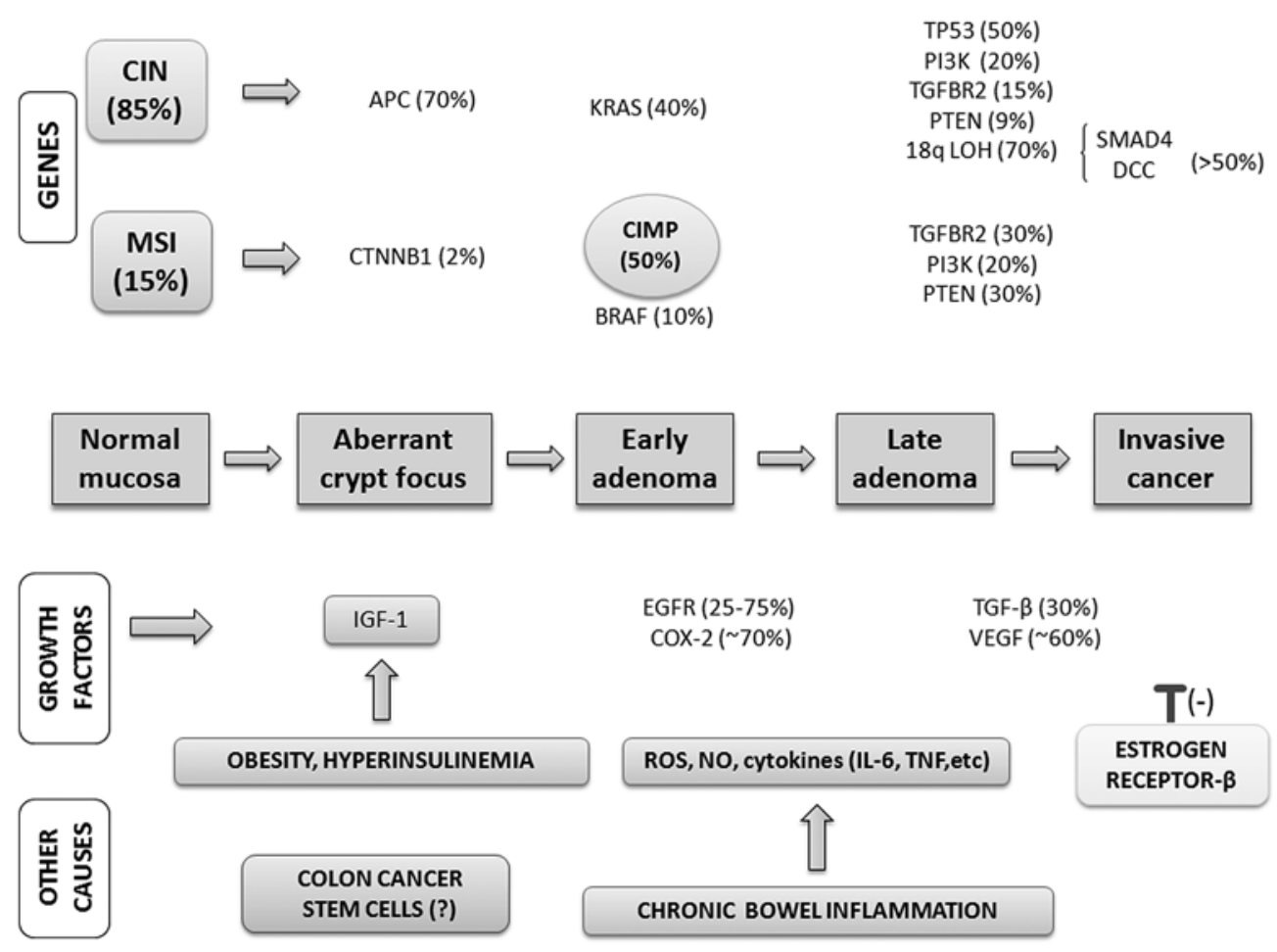

Figure 2. Genes, growth factor pathways and other possibile causes driving multistep colorectal cancer progression. Colorectal carcinogenesis progresses by at least two well-recognized pathways: i) the chromosomal instability (CIN) pathway, which is observed in benign adenomas and increases in tandem with tumor progression; and ii) the microsatellite instability (MSI) pathway, which may be associated with the CpG island methylator phenotype (CIMP) and serrated adenoma pathways. Additionally, growth factor pathways (e.g., mutations in the EGFR pathway, aberrant overexpression of COX-2) are commonly activated in colorectal cancer (CRC). VEGF and TGF- $\beta$ also contribute to colon carcinogenesis, as do lifestyle and dietary factors. Among the latter, obesity and hyperinsulinemia are of particular interest as they increase the levels of bioactive insulin-like growth factor (IGF)-1. Chronic inflammation, in which reactive oxygen and nitrogen species as well as pro-inflammatory cytokines are released, is an important risk factor for CRC in patients with inflammatory bowel diseases. Colon cancers also express type $\beta$ estrogen receptors, which are thought to account for the inhibitory effect of estrogens on tumor growth, based on the inverse relationship between tumor progression and the expression of these receptors. The complex pathogenesis of CRC may be regulated by colon cancer stem cells located within the crypt unit, especially during the early stages of colorectal carcinogenesis.

\section{Diagnosis}

The 'gold standard' for the diagnosis of precancerous lesions and carcinoma involving the colon is colonoscopy, which allows direct visualization, a fairly accurate localization, and the opportunity to obtain tissue samples for histologic evaluation. Colonoscopy has a significant impact on CRC incidence and mortality, preventing about $65 \%$ of CRC cases (1). For every $1 \%$ increase in the colonoscopy rate, the risk of death is reduced by $3 \%$ (45). When a complete colonoscopy extended to the ileo-cecal valve is not possible, air-contrast barium enema and virtual colonoscopy are suitable options.

Until the advent of modern colonoscopy, barium enema was considered the mainstay for the detection of large colonic polyps and colon cancer. However, barium enema is neither as sensitive nor as specific as colonoscopy and should be chosen as the initial screening test only for patients in whom colonoscopy has either failed or who are at high risk for complications from the procedure, or when colonoscopy is not available. Barium enema has no role in determining the extent of colonic wall invasion by colon cancer, nor does it provide information on lymph node involvement or distant disease in patients who are at high risk for metastases.

Computed tomographic (CT) colonography (also known as virtual colonoscopy) was introduced into clinical practice in the mid-1990s and is a promising technique for the diagnosis and screening of CRC. As a minimally invasive imaging examination of the entire colon and rectum, it allows polyp detection, the characterization of tumor density and site, and in some settings evaluation of the extra-colonic structures. The risk of test-related complications is also very low, as the perforation rate associated with CT colonography is $0.06-0.08 \%$, compared with $0.1-0.2 \%$ for colonoscopy (46). In terms of colon cancer detection and the measurement of advanced and adenomatous polyps $\geq 10 \mathrm{~mm}$, recent data indicate that CT colonography is comparable to colonoscopy (47), whereas for lesions 5-9 $\mathrm{mm}$ in diameter the accuracy of the CT examination is lower and for lesions $<5 \mathrm{~mm}$ in diameter it is unacceptable (48). Another potential disadvantage of CT colonoscopy is its poor ability to detect non-polypoid, flat lesions. In a screening population, this subset of sessile polyps had an overall prevalence of around 5.8\% (49). Moreover, virtual colonoscopy is only a diagnostic test and patients with polyps of significant size will require therapeutic colonoscopy for subsequent polypectomy. Thus, at present, CT colonoscopy is not used as a colorectal screening technique and the optimal screening intervals have yet to be established (50).

Another endoscopic technique for colorectal screening is flexible sigmoidoscopy, which is limited to the examination of the lower colon tract but requires neither sedation nor intense bowel preparation, both of which are mandatory for colonos- 
copy. While polypectomy is possible during the examination, patients with lesions $>1 \mathrm{~cm}$ should be referred to colonoscopy since additional adenomatous polyps cannot be excluded. The finding at rigid sigmoidoscopy of one or more advanced adenomas is associated with a rate of metachronous colon cancer, especially in the proximal tract, that is about five times higher than in the general population, whereas for individuals with only small, recto-sigmoid tubular adenomas the risk is the same (6). Sigmoidoscopy, followed by colonoscopy if a polyp or tumor is detected, can identify advanced lesions in $70-80 \%$ of the cases and is associated with a 60-80\% reduction in CRC-related mortality. A single sigmoidoscopy screening between the ages of 55 and 64 years reduces the incidence of CRC by 33\% and mortality by $43 \%$ (51).

Several tools are available to support the clinical diagnosis of genetic syndromes. The Amsterdam criteria I were originally developed to identify families with Lynch syndrome but more than $50 \%$ of these families failed to meet these criteria. To increase the sensitivity, the Amsterdam criteria II and the Bethesda guidelines were developed $(52,53)$. Two other approaches for identifying Lynch syndrome are based on MSI evaluation and immunohistochemistry. In the latter, colorectal tumors are evaluated for MMR deficiency using four antibodies, specific for hMLH1, hMSH2, hMSH6 and hPMS2 proteins. The sensitivity of the two methods is comparable (54).

FAP is diagnosed when at least 100 colonic adenomas are identified, although younger individuals with fewer polyps might also be considered positive for this disease. The diagnosis is supported by the finding of extra-colonic lesions (upper gastrointestinal tract polyposis, congenital hypertrophy of the retinal pigment epithelium, epidermoid cysts, osteomas, dental abnormalities, desmoid tumors) and confirmed by the identification of APC mutations in a proband, allowing precise identification of other relatives who are at risk. Attenuated FAP is suspected when $>10$ but $<100$ adenomas are found in a person older than 40-50 years of age. A precise diagnosis is often difficult in a single patient and the polyp numbers vary with this disorder. Attenuated FAP can mimic the typical, fully expressed form, MUTH-associated polyposis or even sporadic polyp development (16).

As discussed above, the typical gastrointestinal lesions in Peutz-Jeghers syndrome are small-bowel, histologically distinctive hamartomatous polyps ( $96 \%$ of patients). Gastric and colonic Peutz-Jeghers polyps are found in approximately 25 and $30 \%$ of cases, respectively. Generally, gastrointestinal symptoms first occur in the teen-age years, including small-bowel obstruction, intususceptions, and bleeding. In general, a clinical diagnosis of Peutz-Jeghers syndrome is made when two or more of the following features are detected: i) $\geq 2$ Peutz-Jeghers polyps of the small intestine; ii) typical muco-cutaneous hyper-pigmentation; and iii) a family history of the disease (55).

Unlike Peutz-Jeghers syndrome, the physical findings in juvenile polyposis syndrome are not necessarily diagnostic. The key feature of the disease is the occurrence of multiple polyps, most prominently in the colon but also in the stomach, duodenum, and small bowel. A diagnosis of juvenile polyposis should be considered for any young individual of about 20 years with at least three polyps of the colon, multiple polyps throughout the gastrointestinal tract, or any number of polyps and a positive family history. Congenital defects occur in approximately $15 \%$ of patients with juvenile polyposis syndrome. A subset of patients also has hereditary hemorrhagic telangiectasia, often accompanied by mucocutaneous telangiectasias as well as gastrointestinal and pulmonary arteriovenous malformations (56).

A clinical diagnosis of hyperplastic polyposis syndrome is based on at least one of the following criteria: i) $\geq 20-30$ hyperplastic polyps throughout the colon; ii) $\geq 5$ hyperplastic polyps proximal to the sigmoid colon, with two polyps $>1 \mathrm{~cm}$ in diameter; iii) $>1$ hyperplastic polyps proximal to the sigmoid colon; and iv) a first-degree relative with hyperplastic polyposis. Chromo-endoscopy and narrow-band imaging may improve the detection rates of this syndrome (13).

\section{Screening and surveillance}

Both colorectal premalignant and overt malignant lesions are usually asymptomatic and their development is highly insidious. Consequently, screening is often necessary to detect preneoplastic lesions and CRC in its early stages. CRC screening tests are subdivided into two groups: those capable of detecting both cancer and precancerous lesions (structural exams: flexible sigmoidoscopy, colonoscopy, CT colonography, and double-contrast barium enema) and those that primarily detect cancer (stool tests). Colonoscopy remains the most important screening method and has the longest rescreening interval of all diagnostic tests; if normal, the exam does not need to be repeated for 10 years in average-risk adults age 50 years and over (46). Fecal-based screening tests are not invasive and no bowel preparation is necessary but they are less likely to detect adenomas and thus do not contribute to cancer prevention. In addition, they have a significantly lower sensitivity than structural tests for inadequate specimen collection or when processing or interpretation is suboptimal. Moreover, if the stool test is positive, colonoscopy is always necessary.

Two fecal tests for the detection of occult blood are currently available: one is guaiac-based and the other is immunochemical. Guaiac-based tests detect blood in the stool through the pseudo-peroxidase activity of heme or hemoglobin, while immunochemical tests recognize human globin. Although cancers and some large polyps bleed intermittently into the intestinal lumen and occult fecal blood tests can detect very small quantities of blood in stool, the reliability of the test results requires annual testing of two to three samples per test. Patients who have a positive occult fecal blood test are referred for colonoscopy to rule out the presence of polyps or cancer. Studies have shown that the regular use of these screening methods reduces the risk of death from CRC by $15-33 \%$ (1), while the incidence of this disease is reduced by approximately $20 \%$ based on the detection of large polyps, which can subsequently be removed by colonoscopy (57).

The stool DNA test is a screening method that takes advantage of what is currently known about the molecular properties of cancer. Cancer tissues and large polyps shed cells that contain altered DNA into the large bowel; these gene mutations can be detected in stool samples analyzed by DNA tests. Although only a one-time collection is necessary, adequate evaluation requires the entire stool specimen ( $30 \mathrm{~g}$ minimum). The first generation of stool DNA tests assayed for the presense of CIN pathway 
markers such as mutations in APC, KRAS and p53, and the MSI marker BAT-26 (58), while more recent versions are capable of detecting hyper-methylated gene markers of the epigenetic pathway. The first version of this test in asymptomatic patients undergoing colonoscopy identified $51 \%$ of cancers and $18 \%$ of advanced cancer-precursor lesions. In newer versions of the test, the sensitivity is greater: 80 and $40 \%$, respectively (59). Therefore, DNA testing is more accurate than occult fecal blood testing and it can be performed at home; however, it is expensive, the appropriate time interval for repeating the test is uncertain, and the potential for cancer prevention is limited because of poor sensitivity in the detection of colorectal adenomas. Moreover, patients with a positive test are referred for colonoscopy (60).

In 2008, the American Cancer Society, in collaboration with the American College of Radiology and the US Multi-Society Task Force on CRC, published consensus guidelines for CRC screening. The recommendations, in addition to emphasizing cancer prevention as the primary goal of screening (46), recognize three categories of patients.

For the first group, comprising individuals at intermediate risk (adults 50 years of age and older), the currently recommended options include colonoscopy every 10 years, an annual fecal-based test, or flexible sigmoidoscopy or CT colonography every 5 years. Among these tests, colonoscopy is the preferred screening modality.

In the second group are individuals at increased risk, i.e., those with a personal history of adenoma as they are at greater risk of recurrent adenomas or CRC development. For low-risk adenomatous polyps (tubular, two or fewer, $<1 \mathrm{~cm}$ in size), colonoscopy should be repeated within 5 years and, if normal, every 5-10 years. For high-risk adenomatous polyps (villous, 3-10 polyps, $\geq 1 \mathrm{~cm}$, high-grade dysplasia), colonoscopy within 3 years and subsequent surveillance colonoscopies within 5 years are recommended. Individuals with more than 10 adenomatous polyps should undergo evaluation for polyposis syndrome, especially if they are under the age of 40 years and have a strong family history. Despite polypectomy of large sessile adenoma, the recurrence rates are high because residual adenoma tissue is often unavoidable. In this group, colonoscopy should be repeated within 2-6 months.

Individuals with a personal history of CRC who have undergone colonic resection with curative intent have a higher risk of recurrence in the 4-5 years following surgery and should therefore undergo repeat colonoscopy at shorter intervals (1-3 years). For those with a first-degree relative with a diagnosis of CRC between 50 and 60 years, colonoscopy is recommended every 5 years starting at 40 years of age. If CRC is diagnosed in a first-degree relative age 60 years or more, colonoscopy is recommended every 5 years starting from 50 years. In patients with IBD, colonoscopy should be performed 8-10 years after the onset of symptoms (usually the time required for $\mathrm{CRC}$ development) and then repeated every 1-2 years.

For individuals with high-risk inherited syndromes, or a genetic or clinical diagnosis of HNPCC, or an increased risk of HNPCC, colonoscopy should be started at age of 20-25 years, or 10 years before the youngest case of CRC occurs in immediate family members, and then repeated every 1-2 years. In addition, genetic testing should be offered to first-degree relatives. For FAP patients, colonoscopy screening should be started at age
10-12 years and repeated annually. Patients with MAP should undergo colonoscopy at the age of 30-35 years and every 3-5 years thereafter. In those with Peutz-Jeghers syndrome, endoscopic screening for colon cancer should be performed initially during the late teen years and every 2-3 years thereafter, whereas for juvenile polyposis syndrome screening is recommended beginning at the age of 15 years, with annual repeat colonoscopy if polyps are initially detected, otherwise every 2-3 years. Although precise surveillance strategies have not been established in hyperplastic polyposis syndrome, regular colonoscopy is recommended every 1-2 years (46).

\section{Treatment}

Colonoscopy with removal of adenomas is an effective strategy for reducing the incidence of CRC and disease mortality by as much as 70 and $60 \%$, respectively, in the general population, especially for patients with left-side tumors (61). However, standard polypectomy techniques are ineffective in dealing with sessile and non-polypoid colorectal lesions; instead, endoscopic mucosal resection is now the treatment of choice for such cases. Endoscopic mucosal resection relies on the detachment of the submucosal layer from the muscularis propria followed by resection between these layers to effectively remove the lesion. While there are limits to this en bloc technique, as it is effective only for lesions with a maximum diameter of $1.5-2 \mathrm{~cm}$, it is a practical approach with low rates of complications and local recurrences (about 7\%) (62).

An improved understanding of the modifiable risk factors may result in additional primary prevention strategies, even if their efficacy has yet to be determined. A recent study found that a healthy lifestyle, i.e., maintaining a normal weight, being physically active at least $30 \mathrm{~min}$ per day, eating a healthy diet, not smoking, and avoiding excessive amounts of alcohol, was associated with a lower risk of CRC (incidence rate ratio 0.89; 95\% confidence interval: $0.82-0.96$ ), with about $25 \%$ of CRCs considered accordingly preventable (63). In addition, several trials have suggested that calcium and vitamin $\mathrm{D}$, antioxidants (selenium, $\beta$-carotene, vitamins $\mathrm{A}, \mathrm{C}$ and $\mathrm{E}$ ), and folic acid supplements can contribute to CRC prevention (64-66).

It is now well established that non-steroidal anti-inflammatory drugs (NSAIDs) can cause adenoma regression in FAP patients (67). Aspirin and NSAIDs have also been shown to modulate several of the early molecular events in the classic adenoma $\rightarrow$ carcinoma sequence, thus preventing malignant transformation (16), as well as adenoma recurrence and the incidence of CRC in the general population, although this effect was only observed in studies in which at least $300 \mathrm{mg}$ aspirin/day was administered, with a follow-up duration longer than 10 years (68). In a recent study, the long-term administration of lower doses (75-300 mg daily) of aspirin on $\mathrm{CRC}$ incidence resulted in no overall risk reduction for rectal cancer. Notably, the benefit was instead greatest for cancers of the proximal colon, which are not effectively prevented by sigmoidoscopy- or colonoscopy-based screening (69). In general, NSAIDs have a moderate chemopreventive effect, decreasing the number of new adenomas by about $40 \%$ and the number of large or histologically advanced adenomas by up to $60 \%$. Despite this level of effectiveness, NSAIDs are 
Table II. Analysis of some important studies regarding the impact on cancer incidence of the current preventive options.

\begin{tabular}{|c|c|c|c|c|c|c|}
\hline Options for prevention & $\begin{array}{l}\text { No. of } \\
\text { patients }\end{array}$ & $\begin{array}{l}\text { Follow-up } \\
\text { (years) }\end{array}$ & $\begin{array}{l}\text { Reduction of } \\
\text { incidence (\%) }\end{array}$ & P-value & $\begin{array}{c}\mathrm{OR} / \mathrm{RR} \\
(95 \% \mathrm{CI})\end{array}$ & Authors/(Refs.) \\
\hline Polypectomy & 4,344 & 10 & $77 \%$ & $<0.001$ & $0.23(0.19-0.27)$ & Brenner et al (62) \\
\hline Lifestyle and dietary factors & 55,487 & 9.9 & $13 \%$ & 0.2 & $0.89(0.82-0.96)$ & Kirkegaard et al (64) \\
\hline Calcium plus vitamin $\mathrm{D}$ & 36,282 & 7 & $10 \%$ & 0.73 & $1.08(0.86-1.34)$ & Manson et al (65) \\
\hline $\begin{array}{l}\text { Antioxidants (selenium, } \\
\beta \text {-carotene, vitamins A, } \\
\mathrm{C} \text { and } \mathrm{E} \text { ) }\end{array}$ & 676,141 & 20 & $10 \%$ & 0.97 & $0.88(0.81-0.96)$ & Park et al (66) \\
\hline Folic acid & 120,852 & 13.3 & $20 \%$ & 0.18 & $0.85(0.74-0.99)$ & Kennedy et al (67) \\
\hline COX-2 inhibitors & 14,033 & 20 & $45 \%$ & 0.01 & $0.76(0.6-0.96)$ & Rothwell et al (71) \\
\hline Hormonal therapy & 1,831 & 15 & $40 \%$ & 0.05 & $0.52(0.38-0.72)$ & Long et al (72) \\
\hline Statins & 1,818 & 16 & $16 \%$ & 0.8 & $0.99(0.86-1.14)$ & Baron et al (73) \\
\hline
\end{tabular}

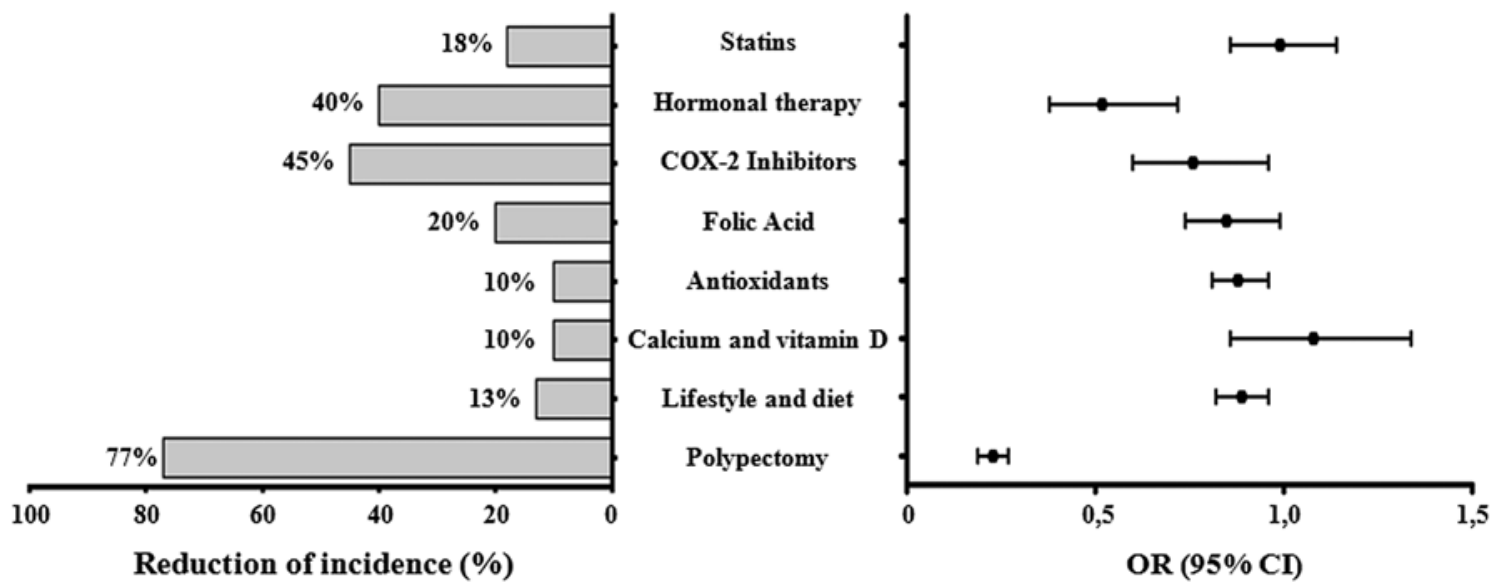

Figure 3. Impact of the current preventive options on the incidence of colorectal cancer.

not currently recommended for adenoma chemoprevention, largely because of their well-known adverse effects. As an alternative, CRC chemoprevention based on combination therapies in patients at high risk of advanced adenomas has gained increasing interest (70).

Although post-menopausal hormone therapy appears to be associated with a lower risk of CRC, it remains unclear which preparations of estrogen-alone or estrogen plus progestin are most effective. A longer duration of treatment is associated with increased protection, although the risk returns to that obtained with placebo within 3 years of hormone cessation. Moreover, because post-menopausal hormones increase the risk of breast cancer and cardiovascular events, the balance of risks and benefits does not support their use as a means of preventing CRC (71). Statins have also been claimed to reduce the risk of $\mathrm{CRC}$, but the evidence has been inconsistent; however, recent studies support the efficacy of statins in preventing the development and progression of adenomatous polyps (72).

Table II and Fig. 3 summarize the impact of the different prevention options on the incidence of colon cancer based on the results of recent studies. Dietary components, lifestyle factors, and medications have been proposed to act either directly or indirectly via anti-inflammatory mechanisms that are largely mediated by COX-2 inhibition, as shown in Fig. 4 (73,74).

In conclusion, only polypectomy currently offers optimal treatment of colorectal preneoplastic lesions and it remains a reliable strategy for CRC prevention, whereas uncertainties remain regarding the effectiveness of lifestyle and dietary factors. The potential toxicity of medications or supplements as protective factors that can reduce CRC development and progression is also a matter of concern.

In inherited syndromes, an awareness of an individual's genetic predisposition and the recognition of the subset of patients at high lifetime risk of CRC allow for a rational patient-tailored clinical management approach that in the future might include effective chemoprevention and biologicsbased treatments. In the meantime, in Lynch syndrome patients, subtotal colectomy with ileo-rectal anastomosis is the recommended approach to colon cancer prevention (75). Since the most common extra-colonic malignancy associated with Lynch syndrome is endometrial cancer (incidence of 40-60\%), with a lifetime risk that is comparable to or even greater than 


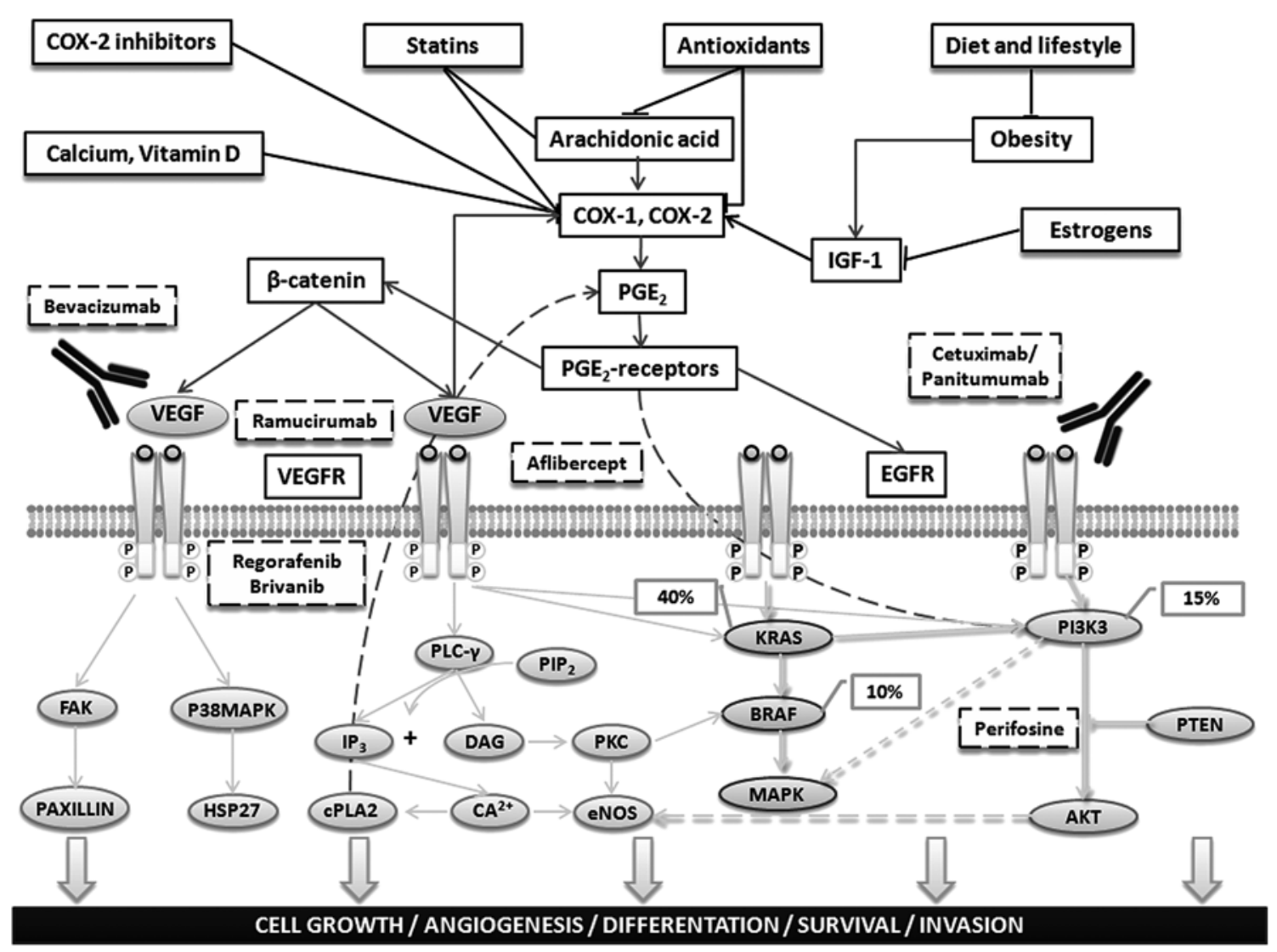

Figure 4. Main preventive and therapeutic agents in colorectal cancer. Dietary components, lifestyle, and medications may exert antineoplastic effects through direct or indirect anti-inflammatory mechanisms that largely result in the inhibition of COX-2. This enzyme is normally upregulated by inflammatory or oncogenic stimuli via inflammatory cytokines, such as interleukin-6 (IL-6), which induce nuclear factor $\kappa \mathrm{B}$ (NF- $\mathrm{\kappa B})$. COX-2 converts membrane-associated arachidonic acid to prostaglandins (PGs) that, in turn, stimulate their respective receptors, resulting in the upregulation of $\beta$-catenin transcriptional activity and activation of the oncogene products phosphatidylinositol-3-kinase (PI3K) and AKT kinase. PGs also trigger phosphorylation of the epidermal growth factor receptor (EGFR), thereby activating PI3K, AKT and the oncogenic RAS-mitogen-activated protein kinase (MAPK) cascade. The EGFR homodimer, formed after ligand activation of the receptor, phosphorylates/activates the intracellular kinase domain and thus also a cascade of downstream signaling that includes activation of the Ras/Raf/MAPK and phosphatidylinositol 3-kinase (PI3K) pathways associated with cell growth, differentiation, survival and invasion. Anti-EGFR monoclonal antibodies (cetuximab, panitumumab) are used to treat patients with metastatic colorectal cancer (CRC). These drugs bind to the extracellular portion of the EGFR to inhibit signaling. Drug resistance can occur via activating mutations, such as in KRAS ( 40\% of CRCs) or its direct downstream effector BRAF ( 10\%), that bypass the need for upstream EGFR signals. Moreover, PGE2-mediated signaling pathways induce the expression of other genes, including vascular endothelial growth factor (VEGF), that have an important role in carcinogenesis by altering normal cellular immunity and apoptosis or by increasing proliferation, angiogenesis, migration, and invasion. Five novel targeted agents for the treatment of CRC that are currently under study in phase III trials are shown.

the estimated risk for CRC, prophylactic hysterectomy and bilateral salpingo-oophorectomy are recommended for female patients after completion of childbearing (76). In patients with FAP, once adenomatous polyps emerge, an annual follow-up colonoscopy is recommended. Colectomy should be considered when more than 20 adenomas develop, when adenomas $>1 \mathrm{~cm}$ in diameter are found, or when advanced histology is diagnosed. Restorative proctocolectomy (also called total proctocolectomy) with ileal pouch anal anastomosis is recommended for patients with large numbers of rectal adenomas. If few or no rectal adenomas are present, then preservation of the rectum with ileo-rectal anastomosis is the preferred surgical therapeutic strategy. Annual or more frequent endoscopic examinations must be performed if any rectal tissue remains. However, up to $33 \%$ of patients with a preserved rectum will eventually require a complete proctectomy because of diffuse polyposis. If numerous adenomas are detected, COX-2 inhibitors should be administered as they are effective in achieving polyp regression, which in turn facilitates surveillance (77).
There are few data on the use of NSAIDs in FAP patients, either to delay surgery or as a primary treatment, and they remain to be tested in investigative trials.

Patients with attenuated FAP should always undergo colonoscopy screening because of the frequency of proximal colonic polyps. Approximately 33\% of these patients can be managed over the long-term with colonoscopy and polypectomy because of the small number of polyps. However, the majority will eventually require colectomy, with rectal-sparing ileo-rectal anastomosis whenever possible. Annual post-operative surveillance is required for polyp ablation, but subsequent proctectomy is rarely needed. Over time, more than $20 \%$ of patients will require therapeutic endoscopic procedures or surgery to treat duodenal adenomas and adenomas at the duodenal papilla. Gastric fundic gland polyps should be sampled, especially if they are large or erythematous. Gastrectomy is needed only if severe dysplasia appears (13).

In MAP, subtotal colectomy is advised for patients who develop colon cancer but it should also be considered when 
colonoscopic management becomes problematic or when polyps become larger or exhibit high-grade dysplasia. Patients with Peutz-Jeghers or juvenile polyposis syndrome should be referred to a specialized center, because of the rarity of these conditions, the complexities of their screening, diagnosis, and management, and the limited data on the effectiveness of various screening modalities (78). In patients with hyperplastic polyposis syndrome, polyps $>5 \mathrm{~mm}$ should be treated by polypectomy. Subtotal colectomy should be considered if colonoscopic treatment is inadequate or high-grade dysplasia occurs (13).

\section{Conclusion}

The remarkable gains in our understanding of the molecular basis of CRC and the transfer of this knowledge into daily clinical practice have begun to reduce the burden of this disease. The mortality rates for CRC are decreasing in several Western countries, and above all in the United States, due to increased awareness and more effective prevention of CRC, the improved early detection of colorectal pre-cancerous lesions, and the introduction of novel, personalized therapeutic options for lowand high-risk individuals.

\section{Acknowledgements}

The study was supported by grants from: Finalized project of the Apulia Region 'Biotecnoter'; Italian Association for Cancer Research; 'Cassa di Risparmio di Puglia' Foundation; University of Bari.

\section{References}

1. American Cancer Society: Colorectal Cancer Facts \& Figures 2011-2013. American Cancer Society Inc., Atlanta, GA, 2011.

2. Jemal A, Bray F, Center MM, et al: Global cancer statistics. CA Cancer J Clin 61: 69-90, 2011.

3. Conteduca V, Sansonno D, Ingravallo G, Marangi S, Russi S, Lauletta G and Dammacco F: Barrett's esophagus and esophageal cancer: an overview. Int J Oncol 41: 414-424, 2012.

4. Conteduca V, Sansonno D, Lauletta G, Russi S, Ingravallo G and Dammacco F: H. pylori infection and gastric cancer: state of the art (Review). Int J Oncol 42: 5-18, 2013.

5. NCCN Clinical Practice Guidelines in Oncology. Colorectal Cancer screening, 2013

6. Levine JS and Ahnen DJ: Adenomatous polyps of the colon. N Engl J Med 355: 2551-2557, 2006.

7. Leggett $B$ and Whitehall V: Role of the serrated pathway in colorectal cancer pathogenesis. Gastroenterology 138: 2088-2100, 2010.

8. Fujita K, Yamamoto H, Matsumoto T, et al: Sessile serrated adenoma with early neoplastic progression: a clinicopathologic and molecular study: Am J Surg Pathol 35: 295-304, 2011.

9. Winawer SJ, Zauber AG, Fletcher RH, et al: Guidelines for colonoscopy surveillance after polypectomy: a consensus update by the US Multi Society Task Force on colorectal cancer and the American Cancer Society. Gastroenterology 130: 1872-1885, 2006.

10. Martínez ME, Baron JA, Lieberman DA, et al: A pooled analysis of advanced colorectal neoplasia diagnoses after colonoscopic polypectomy. Gastroenterology 136: 832-841, 2009.

11. Rizzo A, Pallone F, Monteleone G, et al: Intestinal inflammation and colorectal cancer: a double-edged sword? World J Gastroenterol 17: 3092-3100, 2011.

12. Butterworth AS, Higgins JP and Pharoah P: Relative and absolute risk of colorectal cancer for individuals with a family history: a meta-analysis. Eur J Cancer 42: 216-227, 2006.
13. Jasperson KW, Tuohy TM, Neklason DW, et al: Hereditary and familial colon cancer. Gastroenterology 138: 2044-2058, 2010.

14. Lynch HT and de la Chapelle A: Hereditary colorectal cancer. N Engl J Med 348: 919-932, 2003.

15. Stoffel E, Mukherjee B, Raymond VM, et al: Calculation of risk of colorectal and endometrial cancer among patients with Lynch syndrome. Gastroenterology 137: 1621-1627, 2009.

16. Galiatsatos P and Foulkes WD: Familial adenomatous polyposis. Am J Gastroenterol 101: 385-398, 2006.

17. Lubbe SJ, Di Bernardo MC, Chandler IP, et al: Clinical implications of the colorectal cancer risk associated with MUTYH mutation. J Clin Oncol 27: 3975-3980, 2009.

18. McGarrity TJ and Amos C: Peutz-Jeghers syndrome: clinicopathology and molecular alterations. Cell Mol Life Sci 63: 2135-2144, 2006.

19. Brosens LA, van Hattem A, Hylind LM, et al: Risk of colorectal cancer in juvenile polyposis. Gut 56: 965-967, 2007.

20. Boparai KS, Mathus-Vliegen EM, Koornstra JJ, et al: Increased colorectal cancer risk during follow-up in patients with hyperplastic polyposis syndrome: a multicentre cohort study. Gut 59: 1098-1100, 2010.

21. Alexander DD, Weed DL, Cushing CA, et al: Meta-analysis of prospective studies of red meat consumption and colorectal cancer. Eur J Cancer Prev 20: 293-307, 2011.

22. Park JY, Mitrou PN, Dahm CC, et al: Baseline alcohol consumption, type of alcoholic beverage and risk of colorectal cancer in the European Prospective Investigation into Cancer and Nutrition-Norfolk study. Cancer Epidemiol 33: 347-354, 2009.

23. Kant P and Hull MA: Excess body weight and obesity - the link with gastrointestinal and hepatobiliary cancer. Nat Rev Gastroenterol Hepatol 8: 224-238, 2011

24. Giouleme O, Diamantidis MD and Katsaros MG: Is diabetes a causal agent for colorectal cancer? Pathophysiological and molecular mechanisms. World J Gastroenterol 17: 444-448, 2011.

25. Jin P, Lu XJ, Sheng JQ, et al: Estrogen stimulates the expression of mismatch repair gene hMLH1 in colonic epithelial cells. Cancer Prev Res 3: 910-916, 2010.

26. Ullman TA and Itzkowitz SH: Intestinal inflammation and cancer. Gastroenterology 140: 1807-1816, 2011.

27. Gallimore AM and Godkin A: Epithelial barriers, microbiota, and colorectal cancer. N Engl J Med 368: 3, 2013.

28. Markowitz SD and Bertagnolli MM: Molecular basis of colorectal cancer. N Engl J Med 361: 2449-2460, 2009.

29. Pino MS and Chung DC: The chromosomal instability pathway in colon cancer. Gastroenterology 138: 2059-2072, 2010.

30. Hewish M, Lord CJ, Martin SA, et al: Mismatch repair deficient colorectal cancer in the era of personalized treatment. Nat Rev Clin Oncol 7: 197-208, 2010.

31. Ligtenberg MJ, Kuiper RD, Chan TL, et al: Heritable somatic methylation and inactivation of MSH2 in families with Lynch syndrome due to deletion of the 3 ' exons of TACSTD1. Nat Genet 41: 112-117, 2009.

32. Pritchard CC and Grady WM: Colorectal cancer molecular biology moves into clinical practice. Gut 60: 116-129, 2011.

33. Weisenberger DJ, Siegmund KD, Campan M, et al: $\mathrm{CpG}$ island methylator phenotype underlies sporadic microsatellite instability and is tightly associated with BRAF mutation in colorectal cancer. Nat Genet 38: 787-793, 2006.

34. Hinoue T, Weisenberger DJ, Pan F, et al: Analysis of the association between CIMP and BRAF in colorectal cancer by DNA methylation profiling. PLoS One 4: e8357, 2009.

35. Powell S, Zilz N, Beazer-Barclay Y, et al: APC mutations occur early during colorectal tumorigenesis. Nature 359: 235-237, 1992.

36. Cottrell S, Bicknell D, Kaklamanis L, et al: Molecular analysis of APC mutations in familial adenomatous polyposis and sporadic colon carcinomas. Lancet 340: 626-630, 1992.

37. Nieuwenhuis MH and Vasen HF: Correlations between mutation site in APC and phenotype of familial adenomatous polyposis (FAP): a review of the literature. Crit Rev Oncol Hematol 61: 153-161, 2007.

38. Rennert G, Almog R, Tomsho LP, et al: Colorectal polyps in carriers of the APC I1307K polymorphism. Dis Colon Rectum 48: 2317-2321, 2005.

39. Kim YS and Deng G: Epigenetic changes (aberrant DNA methylation) in colorectal neoplasia. Gut Liver 1: 1-11, 2007.

40. Goss $\mathrm{KH}$ and Groden J: Biology of the adenomatous polyposis coli tumor suppressor. J Clin Oncol 18: 1967-1979, 2000. 
41. Greenhough A, Smartt HJ, Moore AE, et al: The COX-2/PGE2 pathway: key roles in the hallmarks of cancer and adaptation to the tumour microenvironment. Carcinogenesis 30: 377-386, 2009.

42. Vogelstein B, Fearon ER, Hamilton SR, et al: Genetic alterations during colorectal-tumor development. N Engl J Med 319: 525-532, 1988

43. Todaro M, Francipane MG, Medema JP, et al: Colon cancer stem cells: promise of targeted therapy. Gastroenterology 138 : 2151-2162, 2010

44. Zeki SS, Graham TA and Wright NA: Stem cells and their implications for colorectal cancer. Nat Rev Gastroenterol Hepatol 8: 90-100, 2011.

45. Rabeneck L, Paszat LF, Saskin R, et al: Association between colonoscopy rates and colorectal cancer mortality. Am J Gastroenterol 105: 1627-1632, 2010.

46. Levin B, Lieberman DA, McFarland B, et al: Screening and surveillance for the early detection of colorectal cancer and adenomatous polyps, 2008: a joint guideline from the American Cancer Society, the US Multi-Society Task Force on Colorectal Cancer, and the American College of Radiology. Gastroenterology 134: 1570-1595, 2008.

47. Johnson CD, Chen MH, Toledano AY, et al: Accuracy of CT colonography for detection of large adenomas and cancers N Engl J Med 359: 1207-1217, 2008.

48. Laghi A, Iafrate F, Rengo M, et al: Colorectal cancer screening: The role of CT colonography. World J Gastroenterol 16 : 3987-3994, 2010

49. Pickhardt PJ and Kim DH: Performance of CT colonography for detecting small, diminutive, and flat polyps. Gastrointes Endosc Clin N Am 20: 209-226, 2010.

50. Atkin WS, Edwards R, Kralj-Hans I, et al: Once-only flexible sigmoidoscopy screening in prevention of colorectal cancer: a multicentre randomised controlled trial. Lancet 375: 1624-1633, 2010.

51. Hewitson P, Glasziou P, Watson E, et al: Cochrane systematic review of colorectal cancer screening using the fecal occult blood test (hemoccult): an update. Am J Gastroenterol 103: 1541-1549, 2008.

52. Vasen HF, Watson P, Mecklin JP, et al: New clinical criteria for hereditary nonpolyposis colorectal cancer (HNPCC, Lynch syndrome) proposed by the International Collaborative group on HNPCC. Gastroenterology 116: 1453-1456, 1999.

53. Umar A, Boland CR, Terdiman JP, et al: Revised Bethesda guidelines for hereditary nonpolyposis colorectal cancer (Lynch syndrome) and microsatellite instability. J Natl Cancer Inst 96: 261-268, 2004

54. Berg AO, Armstrong K, Botkin J, et al: Recommendations from the EGAPP Working Group: genetic testing strategies in newly diagnosed individuals with colorectal cancer aimed at reducing morbidity and mortality from Lynch syndrome in relatives. Evaluation of Genomic Applications in Practice and Prevention (EGAPP) Working Group. Genet Med 11: 35-41, 2009.

55. Beggs AD, Latchford AR, Vasen HF, et al: Peutz-Jeghers syndrome: a systematic review and recommendations for management. Gut 59: 975-986, 2010.

56. Brosens LA, Langeveld D and van Hattem WA: Juvenile polyposis syndrome. World J Gastroenterol 17: 4839-4844, 2011.

57. Imperiale $\mathrm{TF}$, Ransohoff $\mathrm{DF}$, Itzkowitz $\mathrm{SH}$, et al: Fecal DNA versus fecal occult blood for colorectal-cancer screening in an average-risk population. N Engl J Med 351: 2704-2714, 2004.

58. Ahlquist DA: Next-generation stool DNA testing: expanding the scope. Gastroenterology 136: 2068-2073, 2009.
59. Ahlquist DA, Sargent DJ, Loprinzi CL, et al: Stool DNA and occult blood testing for screen detection of colorectal neoplasia. Ann Intern Med 149: 441-450, 2008.

60. Van Gossum A Munoz-Navas M, Fernandez-Urien I, et al: Capsule endoscopy versus colonoscopy for the detection of polyps and cancer. N Engl J Med 361: 264-270, 2009.

61. Brenner H, MD, Chang-Claude J, Seiler CM, et al: Protection from colorectal cancer after colonoscopy. A population-based, case-control study. Ann Intern Med 154: 22-30, 2011.

62. Ferrara F, Luigiano C, Ghersi S, et al: Efficacy, safety and outcomes of 'inject and cut' endoscopic mucosal resection for large sessile and flat colorectal polyps. Digestion 82: 213-220, 2010.

63. Kirkegaard H, Johnsen NF, Christensen J, et al: Association of adherence to lifestyle recommendations and risk of colorectal cancer: a prospective Danish cohort study. BMJ 341: c5504, 2010.

64. Manson JE, Mayne ST and Clinton SK: Vitamin D and prevention of cancer-ready for prime time? N Engl J Med 364: 1385-1387, 2011.

65. Park Y, Spiegelman D, Hunter DJ, et al: Intakes of vitamins A, $\mathrm{C}$, and $\mathrm{E}$ and use of multiple vitamin supplements and risk of colon cancer: a pooled analysis of prospective cohort studies. Cancer Causes Control 21: 1745-1757, 2010.

66. Kennedy DA, Stern SJ, Moretti M, et al: Folate intake and the risk of colorectal cancer: a systematic review and meta-analysis. Cancer Epidemiol 35: 2-10, 2011

67. Chan AT: Aspirin and familial adenomatous polyposis: coming full circle. Cancer Prev Res (Phila) 4: 623-627, 2011.

68. Cole BF, Logan RF, Halabi S, et al: Aspirin for chemoprevention of colorectal adenomas: meta-analysis of the randomised trials. J Natl Cancer Inst 101: 256-266, 2009.

69. Tsujii M: Search for novel target molecules for the effective treatment or prevention of colorectal cancer. Digestion 85: 99-102, 2012.

70. Rothwell PM, Wilson M, Elwin CE, et al: Long-term effect of aspirin on colorectal cancer incidence and mortality: 20-year follow-up of five randomised trials. Lancet 376: 1741-1750, 2010.

71. Long MD, Martin CF, Galanko JA, et al: Hormone replacement therapy, oral contraceptive use and distal large bowel cancer: a population-based case-control study. Am J Gastroenterol 105: 1843-1850, 2010

72. Baron JA: Statins and the colorectum: hope for chemoprevention? Cancer Prev Res 3: 573-575, 2010.

73. Chan AT and Giovannucci EL: Primary prevention of colorectal cancer. Gastroenterology 138: 2029-2043, 2010.

74. Chu E: An update on the current and emerging targeted agents in metastatic colorectal cancer. Clin Colorectal Cancer 11: 1-13, 2012.

75. Parry S, Win AK, Parry B, et al: Metachronous colorectal cancer risk for mismatch repair gene mutation carriers: the advantage of more extensive colon surgery. Gut 60: 950-957, 2011.

76. Obermair A, Youlden DR, Young JP, et al: Risk of endometrial cancer for women diagnosed with HNPCC-related colorectal carcinoma. Int J Cancer 127: 2678-2684, 2010.

77. Arber N, Spicak J, Rácz I, et al: Five-year analysis of the prevention of colorectal sporadic adenomatous polyps trial. Am J Gastroenterol 106: 1135-1346, 2011.

78. Bolocan A, Ion D, Stoian RV, et al: MAP syndrome (MYH associated polyposis) colorectal cancer, etiopathological connections. J Med Life 4: 109-111, 2011. 